\title{
Differentially Coherent Code Acquisition in the Multiple Transmit/Receive Antenna Assisted Multi-Carrier DS-CDMA Downlink
}

\author{
SeungHwan Won and Lajos Hanzo \\ School of ECS, Univ. of Southampton, SO17 1BJ, UK. \\ Tel: +44-23-8059 3125, Fax: +44-23-8059 4508 \\ E-mail:\{shw04r, lh\}@ecs.soton.ac.uk \\ http://www-mobile.ecs.soton.ac.uk
}

\begin{abstract}
We examine both differentially coherent and non-coherent code acquisition schemes designed for the multiple transmit/receive antenna assisted Multi-Carrier (MC)-DS-CDMA downlink, when communicating over uncorrelated Rayleigh channels. It is demonstrated that in contrast to our expectations, when the number of transmit antennas and/or that of the subcarriers is increased in both the differentially coherent and the non-coherent code acquisition scenarios, the achievable Mean Acquisition Time (MAT) usually deteriorates over the entire Signal-to-Interference plus Noise Ratio (SINR) per chip $\left(E_{c} / I_{0}\right)$ range considered.
\end{abstract}

\section{INTRODUCTION}

Employing both multiple transmit antennas and subcarriers in the downlink of wireless systems exhibits an attractive technique of reducing the detrimental effects of time-variant multi-path fading environments [1]-[3]. In recent years diverse combinations of SingleCarrier (SC) CDMA and OFDM [4] have attracted research efforts [2]-[4]. In inter-cell synchronous CDMA systems the mobile station's receiver must be capable of synchronising the timing of a locally generated PseudoNoise (PN) spreading sequence with that of the desired user's PN sequence contaminated by the interfering signals. The code acquisition performance of MC DS-CDMA attained with the aid of serial search based schemes has been investigated in [3],[5]. In order to characterise the effects of multiple transmit/receive antennas in terms of the achievable MAT performance, the results of [6] outlined the characteristics of a serial search based code acquisition scheme in the context of the multiple transmit antenna aided SC-DS-CDMA downlink. However, since there is a paucity of in-depth results on the fundamental characteristics of code acquisition schemes designed for a multiple transmit/receive antenna assisted MCDS-CDMA system in the context of Differentially Coherent (DC) code acquisition schemes, solving this open problem is the main objective of the present paper. Similarly to Non-Coherent (NC) code acquisition schemes [5],[6], no prior information concerning the absolute carrier phase is necessary for the operation of DC code acquisition schemes [7]. An additional benefit of using a DC code acquisition scheme is that it is capable of attaining a better performance than its NC counterpart [5]-[7]. Here we adopted the Full-Period Correlation (FPC) based scheme of [7] in order to analyse the characteristics of serial search based DC code acquisition in the multiple transmit/receive antenna assisted MC-DS-CDMA downlink. Against this backdrop, in this treatise we examine both serial search based DC and NC code acquisition schemes designed for multiple transmit/receive antenna assisted MC-DS-CDMA systems. More explicitly, we characterise the MAT versus $E_{c} / I_{0}$ performance parameterised by both the number of transmit/receive antennas and that of the subcarriers.

The financial support of the Ministry of Information and Communication(MIC), Republic of Korea and the European Union under the auspices of the Phoenix and Newcom projects and that of the EPSRC is gratefully acknowledged.
This paper is organised as follows. The MAT expressions of code acquisition designed for multiple transmit/receive antenna assisted MC-DS-CDMA systems are introduced in Section II. Section III describes the system investigated, followed by the correct detection and false alarm probability analysis of the DC code acquisition scheme considered in the context of uncorrelated Rayleigh channels. In Section IV, our numerical MAT results are discussed, while our conclusions are detailed in Section V.

\section{MAT ANALYSIS OF CODE ACQUISITION}

In the case of code acquisition contrived for both MC-DS-CDMA and SC-DS-CDMA systems, the main design goal is to acquire perfect timing of the first received signal path impinging at the receiver, since this timing information is used as that of the reference finger of the Rake receiver. Then the accurate timing positions of the remaining delayed paths are extracted by the post-initial acquisition procedure in [6]. Here we focus our attention on the achievable MAT performance of the first received path. Explicit MAT formulae derived for a single-antenna assisted serial search based code acquisition arrangement were provided in [8]. There is no difference between a single-antenna and a multiple-antenna aided MC-DS-CDMA system in terms of characterising their MAT performance, except for the derivation of the correct detection and the false alarm probability as a function of both the number of transmit/receive antennas and that of the subcarriers. We will continue our discourse here by comparing the MAT performance of DC FPC based code acquisition to that of its NC counterpart employing Single Dwell Serial Search (SDSS) [8], because the correlation operation of the FPC scheme is carried out over a full PN code period [7]. We postulate that in each chip duration $T_{c}, l$ number of correct timing hypotheses are examined, which are spaced by $T_{c} / l$. Hence the entire uncertainty region is increased $l$-fold. All the resultant $(\nu-2 l)$ number of states that may lead to a false alarm are expected to increase the MAT according to the corresponding penalty time. The $2 l$ legitimate locking states within a lag of one chip duration of the correct timing instant are taken into account in the MAT analysis. Combining all these $2 l$ legitimate locking states into the correct detection transfer function encompassing all branches of the relevant state diagram in Fig.3.4 of [8] leads to the correct detection transfer function expressed as

$$
H_{D}(z)=\sum_{j=1}^{2 l} P_{D j} z \prod_{i=1}^{j-1}\left[\left(1-P_{D i}\right) z\right]
$$

where $P_{D j}$ represents the correct detection probability associated with the $j^{\text {th }}$ correct detection, following $(j-1)$ trials each resulting in a miss and where each probability of a miss in the corresponding total miss transfer function is given by $\left(1-P_{D i}\right)$. Furthermore, $z$ indicates the unit-delay operator and the exponent of $z$ represents the time delay, while $H_{0}(z)$ of Fig.3.3 in [8] denotes the absence of the 
desired user's signal at the output of the acquisition scheme, which is expressed as

$$
H_{0}(z)=\left(1-P_{F}\right) z+P_{F} z^{K+1},
$$

where $P_{F}$ represents the false alarm probability and $K$ is the false locking penalty factor [8]. Finally, $H_{M}(z)$ represents the overall miss probability of a search run carried out across the entire uncertainty region, which may be formulated as the product of the individual miss probabilities, since these may be considered independent events, yielding

$$
H_{M}(z)=z^{2 l} \prod_{j=1}^{2 l}\left(1-P_{D j}\right) .
$$

Then, it may be shown that the generalised expression formulated for calculating the MAT of the serial search based code acquisition scheme is given by [8]:

$$
\begin{array}{r}
E\left[T_{A C Q}\right]=\frac{1}{H_{D}(1)}\left[H_{D}{ }^{\prime}(1)+H_{M}{ }^{\prime}(1)+\right. \\
\left.\left\{(\nu-2 l)\left[1-\frac{H_{D}(1)}{2}\right]+\frac{1}{2} H_{D}(1)\right\} H_{0}{ }^{\prime}(1)\right] \cdot \tau_{D},
\end{array}
$$

where $\left.H_{x}^{\prime}(z)\right|_{x=D, M}$ or 0 represents a derivative of $\left.H_{x}(z)\right|_{x=D, M \text { or } 0}$ and $\tau_{D}$ represents the integral dwell time.

\section{THEORETICAL ANALYSIS OF DIFFERENTIALLY COHERENT CODE ACQUISITION}

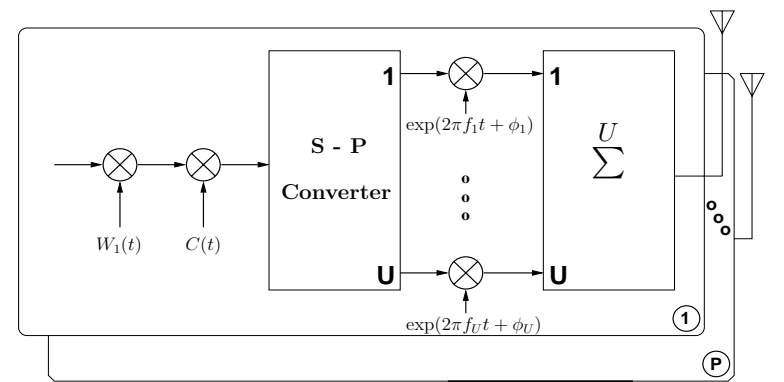

Fig. 1. Transmitter schematic of the MC-DS-CDMA downlink having $P$ transmit antennas and $U$ subcarriers.

Fig. 1 portrays the transmitter schematic of the MC-DS-CDMA downlink having $P$ transmit antennas and $U$ subcarriers. In the MC-DS-CDMA system considered the input bit sequence is Serialto-Parallel (SP) converted and each of the parallel sequences is transmitted on a separate subcarrier, as seen in Fig.1. Furthermore, $T_{b}$ indicates the bit duration of the data sequence before SP conversion, whilst $T_{s}$ represents the symbol duration after SP conversion. Consequently, we have $T_{s}=U \cdot T_{b}$. The total allocated power is equally shared by both the $P$ transmit antennas and $U$ subcarriers as well as $S F=T_{s} / T_{c}$ denotes the spreading factor of the subcarrier signals in the MC-DS-CDMA system, whilst the spreading factor of a corresponding identical-bandwidth SC-DS-CDMA system is $S F_{1}=$ $T_{b} / T_{c 1}$, where $T_{c 1}$ represents the chip duration of the corresponding SC-DS-CDMA signal. For simplicity, in our forthcoming discourse we assume that there is no overlap between the main spectral lobes of two adjacent subcarriers in the MC-DS-CDMA system considered here [5]. Furthermore, we postulate that each subcarrier signal occupies an identical bandwidth and the total bandwidth is equally divided among the $U$ number of subcarriers. Hence, the relationships of $T_{c}=U \cdot T_{c 1}$ and $S F_{1}=S F$ hold, since we have
$T_{s}=U \cdot T_{b}$. The above assumptions allow their direct comparison in our forthcoming analysis. Further relationships between the MC-DSCDMA and the SC-DS-CDMA systems can be found in [5].

The transmitted signal of the multiple transmit antenna assisted base station can be expressed as

$$
\begin{array}{r}
S_{\text {tot }}(t)=\sum_{p=1}^{P} \sum_{u=1}^{U}\left[\sqrt{\frac{E_{c}}{P T_{c}}} b(t) C(t) W_{p}(t)\right. \\
\left.\cdot \exp \left(2 \pi f_{u} t+\phi_{u}\right)\right],
\end{array}
$$

where $p=1, \ldots, P$ indicates the number of transmit antennas, $u=$ $1, \ldots, U$ is the number of subcarriers, $b(t)$ represents the pilot data sequence assuming a value of binary ' 1 ' [8], $C(t)$ denotes the unique user-specific PN sequence, $W_{p}(t)$ identifies the specific Walsh code assigned to the $p^{t h}$ transmit antenna, $E_{c}$ denotes the pilot signal energy per PN code chip, $T_{c}$ indicates the chip duration, $f_{u}$ is the $u^{\text {th }}$ subcarrier frequency, and $\phi_{u}$ denotes the $u^{t h}$ subcarrier phase of the modulator. The received signal of the MIMO-assisted MC-DS-

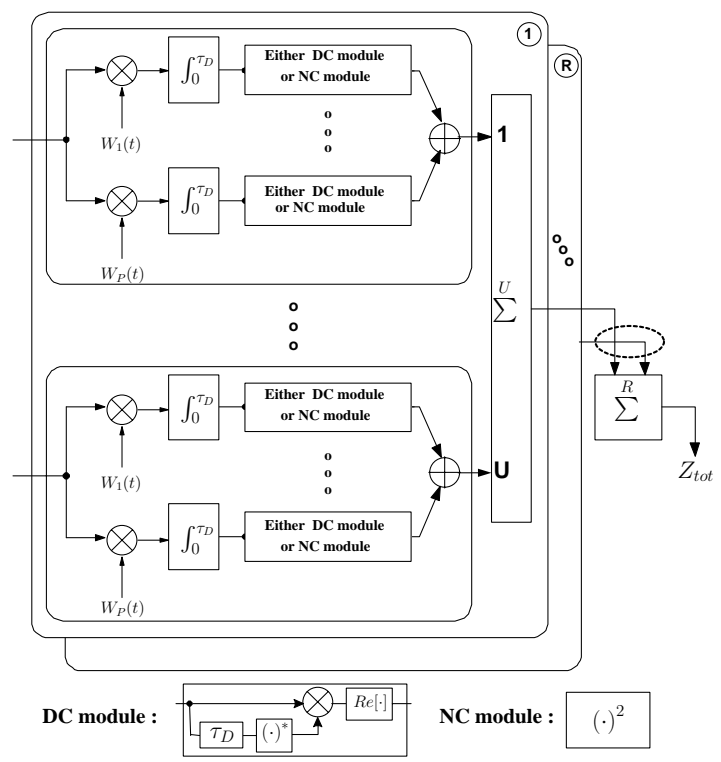

Fig. 2. Receiver structure of both differentially coherent and non-coherent code acquisition employing both $R$ receive antennas and $U$ subcarriers.

CDMA downlink may be written as

$$
\begin{array}{r}
r_{\text {tot }}(t)=\sum_{p=1}^{P} \sum_{r=1}^{R} \sum_{u=1}^{U}\left[\alpha_{(p, r, u)} \sqrt{\frac{E_{c}}{P T_{c}}} C\left(t+d T_{c}\right) W_{p}\left(t+d T_{c}\right)\right. \\
\left.\cdot \exp \left(2 \pi f_{u} t+\phi_{(p, r, u)}\right)+I_{(p, r, u)}(t)\right],
\end{array}
$$

where $r=1, \ldots, R$ indicates the number of receive antennas, $\alpha_{(p, r, u)}$ is the envelope of the $(p, r, u)^{t h}$ received signal path obeying the Rayleigh distribution, $d$ indicates the code phase offset with respect to the phase of the local code, and $\phi_{(p, r, u)}$ is the subcarrier phase of the modulator at the $(p, r, u)^{t h}$ path. Furthermore, $I_{(p, r, u)}(t)$ is the complex-valued Additive White Gaussian Noise (AWGN) having a double-sided power spectral density of $I_{0}$, which contaminates the $(p, r, u)^{t h}$ path. Fig. 2 portrays both the DC and the NC receiver's schematic designed for our code acquisition scheme using multiple transmit/receive antennas, where the NC module generates its decision variable by accumulating $(P \cdot R \cdot U)$ number of independently faded signals observed over a given time interval. In the DC module of Fig.2, instead of squaring the summed energy as suggested by 
the procedures outlined in [6],[8], the channel's output samples accumulated over a full spreading code period are multiplied by the conjugate of the $N$-chip-delayed samples [7]. Due to space limitations here, we omitted formulating the final decision variable, which may be readily derived using the procedures proposed in [7] in the context of the receiver structure of Fig.2. The final DC decision variable may be expressed as [7]

$$
\begin{aligned}
& Z_{k}^{d c}=\sum_{p=1}^{P} \sum_{r=1}^{R} \sum_{u=1}^{U}\left[\left(\sqrt{\frac{4 E_{c}}{N I_{0} P}} \cdot S_{k(p, r, u)}+W_{1, k(p, r, u)}\right)^{2}\right. \\
& \left.+W_{3, k(p, r, u)}^{2}\right]-\sum_{p=1}^{P} \sum_{r=1}^{R} \sum_{u=1}^{U}\left[W_{2, k(p, r, u)}^{2}+W_{4, k(p, r, u)}^{2}\right],
\end{aligned}
$$

where $k$ indicates the $k^{\text {th }}$ chip's sampling instant, $S_{k(p, r, u)}$ is assumed to be deterministic [7] and the definition of $W_{1, k(p, r, u)}$, $W_{2, k(p, r, u)}, W_{3, k(p, r, u)}$ and $W_{4, k(p, r, u)}$ is the same as in [7], which are mutually independent Gaussian random variables having zero means and unit variances [7]. For the simplicity of the derivation let us now introduce a shorthand for the first and second terms of Eq.7 as $X_{k}$ and $Y_{k}$, respectively. Then the final decision variable of Eq.7 is expressed as $Z_{k}^{d c}=X_{k}-Y_{k},=\sum_{p=1}^{P} \sum_{r=1}^{R} \sum_{u=1}^{U} X_{k(p, r, u)}-$ $\sum_{p=1}^{P} \sum_{r=1}^{R} \sum_{u=1}^{U} Y_{k(p, r, u)}$, where $X_{k}$ is noncentrally chi-square distributed with $(2 P \cdot R \cdot U)$ degrees of freedom and its noncentrality parameter $\lambda_{x}$ is either $\frac{4 N}{P}\left(\frac{E_{c}}{I_{0}}\right)^{\prime}$ for the hypothesis of the desired signal being present $(x=1)$ or $\frac{4}{N P}\left(\frac{E_{c}}{I_{0}}\right)^{\prime}$ for it being absent $(x=0)$ [7], while $Y_{k}$ follows a central chi-square PDF with $(2 P \cdot R \cdot U)$ degrees of freedom. In the spirit of [8], the definition of $\left(E_{c} / I_{0}\right)^{\prime}$ encapsulates the effects of both timing errors and frequency mismatches. Accordingly, the PDF of $X_{k}$ and $Y_{k}$ can be expressed as [9]

$$
\begin{array}{r}
f_{X_{k}}\left(z \mid H_{x}\right)=\frac{1}{2}\left(\frac{z}{\lambda_{x}}\right)^{\frac{(P R U-1)}{2}} \cdot \exp \left[-\frac{\left(z+\lambda_{x}\right)}{2}\right] \\
\cdot \mathcal{I}_{(P R U-1)}\left(\sqrt{z \cdot \lambda_{x}}\right)
\end{array}
$$

and

$$
f_{Y_{k}}\left(z \mid H_{x}\right)=\frac{1}{2^{P R U} \cdot \Gamma(P R U)} \cdot z^{(P R U-1)} \cdot \exp \left[-\frac{z}{2}\right],
$$

respectively, where we have $z \geq 0, x=0$ or $1, \Gamma(\cdot)$ denotes the Gamma function and $\mathcal{I}_{(P R U-1)}(\cdot)$ is the $(P \cdot R \cdot U-1)^{s t}$-order modified Bessel function. Our objective is now that of deriving the PDF of the desired user's signal conditioned on the presence of the desired signal in $f_{X_{k}}\left(z \mid H_{x}\right)$ formulated for transmission over an uncorrelated Rayleigh channel. Therefore, first the PDF $f_{X_{k(p, r, u)}}\left(z \mid H_{x}, \beta\right)$ corresponding to a specific SINR $\beta$ conditioned on the hypothesis of the desired signal being transmitted over an AWGN channel having this specific SINR is weighted by the probability of occurrence $f(\beta)$ of encountering the SINR $\beta$, as quantified by the PDF. The resultant product is then averaged over its legitimate range of $-\infty \sim \infty$, yielding:

$$
\begin{array}{r}
f_{X_{k(p, r, u)}}\left(z \mid H_{x}\right)=\int_{-\infty}^{\infty} f(\beta) \cdot f_{X_{k(p, r, u)}\left(z \mid H_{x}, \beta\right) d \beta} \\
\equiv \frac{\exp \left[-z /\left(2+\overline{\lambda_{x}}\right)\right]}{\left(2+\overline{\lambda_{x}}\right)}
\end{array}
$$

where the corresponding noncentrality parameter of $\overline{\lambda_{x}} \equiv \lambda_{x} \sigma^{2}$ is either $\frac{4 N}{P}\left(\frac{\overline{E_{c}}}{I_{0}}\right)^{\prime}$ when the desired signal is deemed to be present $(x=1)$ or $\frac{4}{N P}\left(\overline{\frac{E_{c}}{I_{0}}}\right)^{\prime}$ when it is deemed to be absent $(x=0)$. For notational convenience we also define a new biased noncentrality parameter $\mu_{x}=\left(2+\overline{\lambda_{x}}\right)$. Further details on the related derivations are provided in [7],[8]. Finally, we arrive at the PDF of $X_{k(p, r, u)}$ conditioned on the presence of the desired signal in the form of:

$$
f_{X_{k(p, r, u)}}\left(z \mid H_{x}\right)=\frac{1}{\mu_{x}} e^{-z / \mu_{x}} .
$$

By contrast, the PDF of $f_{Y_{k(p, r, u)}}\left(z \mid H_{x}\right)$ may be readily derived from Eq.9, leading to

$$
f_{Y_{k(p, r, u)}}\left(z \mid H_{x}\right)=\frac{1}{2} e^{-z / 2} .
$$

Since the decision variables, $X_{k}$ and $Y_{k}$ are expressed as the sum of $(P \cdot R \cdot U)$ number of independent variables, each of which obeys a PDF given by Eq.12 or Eq.13, respectively. Both decision variables constitute independent Gamma-distributed variables, as mentioned in [8], yielding:

$$
\begin{aligned}
f_{X_{k}}\left(z \mid H_{x}\right) & =\frac{z^{(P R U-1)} e^{-z / \mu_{x}}}{\Gamma(P R U) \cdot \mu_{x}^{P R U}}, \\
f_{Y_{k}}\left(z \mid H_{x}\right) & =\frac{z^{(P R U-1)} e^{-z / 2}}{\Gamma(P R U) \cdot 2^{P R U}} .
\end{aligned}
$$

Then, the PDF of $Z_{k}^{d c}=X_{k}-Y_{k}$ can be calculated by straightforward convolution of the PDFs of both $X_{k}$ and $Y_{k}$, which leads to the PDF of the difference between two independent Gamma variables. To elaborate a little further, we have $X_{t o t} \sim g\left(P \cdot R \cdot U, \mu_{x}\right)$ and $Y_{t o t} \sim g(P \cdot R \cdot U, 2)$, where this shorthand represents that both $X_{\text {tot }}$ and $Y_{\text {tot }}$ obey a Gamma distribution having the shape parameter of $(P \cdot R \cdot U)$ and the scale parameter of either $\mu_{x}$ or 2 , respectively, as outlined in [10]. The convolution of the PDFs $f_{X_{k}}$ and $f_{Y_{k}}$ formulated in order to compute the PDF of $Z_{k}^{d c}$ conditioned on the desired signal being present or absent is derived as [10]. Let us now define the three parameters, namely $a=P \cdot R \cdot U-0.5$, $b=\left(4 \mu_{x}\right) /\left(\mu_{x}+2\right)$ and $c=-\left(\mu_{x}-2\right) /\left(\mu_{x}+2\right)$, which allow us to express the probability of correct detection or false alarm according to $x=1$ or 0 , respectively, as follows [10]:

$$
\begin{array}{r}
\left.P^{d c}\right|_{x=1 \text { or } 0}=\int_{\theta}^{\infty}\left(\frac{\left(1-c^{2}\right)^{a+\frac{1}{2}} \cdot|z|^{a}}{\sqrt{\pi} \cdot 2^{a} \cdot b^{a+1} \cdot \Gamma\left(a+\frac{1}{2}\right)}\right) \\
\left.\cdot \exp \left(-\frac{c}{b} z\right) \cdot K_{a}\left(\frac{|z|}{b}\right) d z\right|_{x=1 \text { or } 0}, \theta \neq 0,
\end{array}
$$

where $K_{a}(\cdot)$ indicates the modified Bessel function of the second kind and of order $a$ and $\theta$ is a threshold value. For comparison, the NC counterpart of the previously described DC scheme is analysed in [6].

\section{NUMERICAL SYSTEM PERFORMANCE RESULTS}

TABLE I

MAXIMUM SINR DEGRADATION INFLICTED BY BOTH THE DOPPLER SHIFT AND A $1000 \mathrm{HZ}$ FREQUENCY MISMATCH ASSOCIATED WITH THE COHERENT INTEGRATION INTERVAL OF $N$ CHIP DURATIONS AT A CARRIER FREQUENCY OF $1.9 \mathrm{GHZ}$ AS A FUNCTION OF THE NUMBER OF SUBCARRIERS ( $U=1$ AND 4$)$

\begin{tabular}{|l|l|l|l|l|l|}
\hline N(Chips): U=1 & 128 & 256 & $\mathbf{5 1 2}$ & 768 & 1024 \\
\hline N(Chips): U=4 & 32 & 64 & $\mathbf{1 2 8}$ & 192 & 256 \\
\hline Degradation $(\mathrm{dB})$ & 0.061 & 0.2449 & $\mathbf{0 . 9 9 6 9}$ & 2.3144 & 4.3213 \\
\hline
\end{tabular}

The numerical MAT performance of the multiple transmit/receive antenna assisted MC-DS-CDMA code acquisition scheme of Fig.2 
will be characterised. Our performance comparison between the SCDS-CDMA system $(U=1)$ and the MC-DS-CDMA system using $U=4$ subcarriers is based upon the assumptions that these systems have the same total transmitted energy per chip. Furthermore, it is assumed that $\tau_{D}$ is the same for all the scenarios considered here. In Table 1 we summarised the maximum SINR degradation inflicted by both the Doppler shift and the frequency mismatch between the transmitter and receiver in conjunction with the coherent integration interval of $\tau_{D}$ duration, as seen in Fig.2. It was assumed that the length of the PN sequence in our system was $128 \cdot T_{c}$ ( or $512 T_{c 1}$ ), where the chip-durations chosen for $U=1$ and 4 are $T_{c 1}=1 / 2.4576 \mu \mathrm{s}$ and $T_{c}=1 / 0.6144 \mu \mathrm{s}$, respectively. The coherent accumulator of Fig.2 adopted the FPC scheme of [7], where accumulation was carried out over 512 chips in the SC scheme of $U=1$ and over 128 chips in the $U=4$ MC-DSCDMA arrangement, respectively. These optimised parameter values were computed by using Eq.4, Eq.16 and Eq.12 of [6] as well as Eq.(3.7) of [8], which were provided for quantifying the performance degradation imposed by both the Doppler shift and the frequency mismatch encountered. The spreading factor of the Walsh code to be acquired was chosen to be 128 . The carrier frequency was $1.9 \mathrm{GHz}$, whilst the frequency mismatch was postulated to be $1000 \mathrm{~Hz}$ [8]. As a worst-case mobile speed, it is reasonable to assume $160 \mathrm{~km} / \mathrm{h}$. We also assumed that the sampling inaccuracy caused by having a finite, rather than infinitesimally low search step size of $\Delta=1 / 2 T_{c}$ was $-0.91 \mathrm{~dB}$, which is a practically acceptable value for the specific search step size considered [8]. The entire uncertainty region of code acquisition was assumed to entail 512 hypotheses, which is the uncertainty region of the $U=2$ scenario, because the same uncertainty region is required for a fair comparison. Finally, in the spirit of [6], the false locking penalty factor was considered to be 1000 chip-duraions. For simplicity, it was assumed that only a single received signal path is encountered in a given search window. All the performance curves have been generated at the threshold value of $E_{c} / I_{0}=-19 \mathrm{~dB}$.

Fig.3 illustrates the achievable MAT versus SINR per chip performance of SDSS for the DC SC-DS-CDMA code acquisition arrangement as a function of the number of transmit antennas for $P=$ 1,2 as well as 4 and that of the number of receive antennas for $R=1$ and 4. Observe in Fig.3 that unexpectedly, as the number of transmit antennas is decreased, all the curves explicitly indicate an improved MAT performance, except for the ' $P 2 R 1^{\prime}$ scenario. To elaborate on the above observation a little further, a useful transmit diversity gain is only experienced for the case of ${ }^{\prime} P 2 R 1^{\prime}$, and even this was limited to the specific SINR range of -13 to $-16 \mathrm{~dB}$. For comparison, Fig.4 characterises the MAT versus SINR per chip performance of SDSS for the NC code acquisition scheme as a function of the number of transmit antennas for $P=1,2$ as well as 4 and that of the number of receive antennas for $R=1$ and 4 . Similarly, as the number of transmit antennas is decreased, all the curves illustrate an improved MAT peformance. Since the DC scheme has a performance gain of just under $3 \mathrm{~dB}$ over the $\mathrm{NC}$ one, when considering their correct detection probability and false alarm probability, hence we conclude that the MAT performance curves confirm the expected trends. Moreover, the DC scheme has an advantage over the NC one in the low SINR range in terms of reducing the effects of both the AWGN and interference. Hence, this indicates that the DC scheme experiences a lower MAT peformance degradation owing to the employment of multiple transmit antennas than its NC counterpart.

Fig.5 and 6 illustrate the achievable MAT versus SINR per chip performance of the SDSS-aided MC-DS-CDMA code acquisition scheme in both DC and NC scenarios, respectively, when parameterised by both the number of transmit and receive antennas and using $U=4$ subcarriers. In the case of the MC-DS-CDMA system, both schemes benefit from a specific diversity order, which is determined by the number of subcarriers used. It is also assumed that the total transmitted energy per chip is the same in all the scenarios considered. Accordingly, the achievable diversity order is determined by the product of the number of subcarriers and that of the number of transmit antennas, as documented in [3],[5],[6]. This phenomenon indicates that the employment of MC transmissions leads to exactly the same detrimental effect on the achievable MAT performance, as that imposed by employing multiple transmit antennas owing to the reduced 'per-diversity-branch' power, which further argued below. The results of Fig. 5 and Fig. 6 are parameterised by both the number of transmit antennas for $P=1,2$ as well as 4 and by the number of receive antennas for $R=1$ as well as 2 . As both the number of transmit antennas and that of the subcarriers is decreased, all the curves of Fig.5 and Fig.6 exhibit an improved MAT peformance. Furthermore, as a benefit of the inherent performance gain of the DC scheme over the NC one, the overall MAT peformance results of Fig.5 are better than those of Fig.6. This trend explicitly illustrates that the SDSS-aided MC-DS-CDMA code acquisition scheme considerably degrades the achievable MAT performance of SC-DS-CDMA. This is a consequence of both the low per-antenna power imposed by using multiple transmit antennas for the sake of achieving either a transmit diversity gain or a multiplexing gain as well as that of the low per-subcarrier power imposed by having multiple subcarriers in order to attain a frequency diversity gain. A low level of per-branch and/or per-subcarrier received signal strength is expected to result in a low acquisition performance, despite achieving a high transmit- and frequency-diversity gain. The main reasons for the above-mentioned phenomenon are multifold:

1) In general, coherently detected space-time-frequency transmission schemes benefit from having explicit knowledge of the Channel's Impulse Response (CIR), which is unavailable during the codeacquisition phase. Furthermore, the MIMO-aided code-acquisition schemes are only capable of achieving a rather limited time diversity, even when a relatively high (namely, 2 to 4 ) number of Post-Detection Integration (PDI) stages is used [8].

2) Since no channel coding is used for the pilot signal, no time diversity gain is achieved [11].

3) When the detection threshold $\theta$ for the system of Fig.2 is reduced, the resultant code phase estimate often cannot be confirmed by the threshold comparison of Fig.2 and hence the false alarm probability is increased. At the same time, the correct detection probability is also increased. However, when aiming for the best achievable MAT performance, the detection threshold optimisation has to strike a balance.

4) The effect of using a fixed threshold of $\theta$, which is optimised for a specific $E_{c} / I_{0}$ value also limits the attainable MAT performance, since the acquisition threshold should be optimised and controlled as a function of the $E_{c} / I_{0}$ value encountered.

\section{CONCLUSION}

In this contribution, surprisingly, our conclusions suggest that increasing both the number of transmit antennas and that of the subcarriers in a MIMO-assisted MC-DS-CDMA system leads to amalgamating the low-energy signals of both the transmit antennas and the subcarriers, which may increase the MAT by an order of 
magnitude, in particular in the critical scenario, when the SINR is relatively low. Consequently our future research will be aimed at specifically designing turbo-like iterative acquisition schemes [3] for MIMO-aided MC transmission systems.

\section{REFERENCES}

[1] D. Gesbert, M. Shafi, D.S. Shiu, P.J. Smith, and A. Naguib, From Theory to Practice: An Overview of MIMO Space-Time Coded Wireless Systems, IEEE Journal on Selected Areas in Communications, vol. 21, No.3, Issue 3, 2003, pp281-302.

[2] L-L Yang, L. Hanzo, Multicarrier DS-CDMA: A Multiple Access Scheme for Ubiquitous Broadband Wireless Communications, IEEE Communications Magazine, Vol. 41, No.10 Issue 10, 2003, pp116124.

[3] L. Hanzo, L-L Yang, E-L Kuan, K. Yen, Single- and Multi-Carrier DS-CDMA, John Wiley \& Sons, 2003, pp1077.

[4] L. Hanzo, M.Munster, B.J. Choi and T.Keller, OFDM and MCCDMA for Broadcasting Multi-User Communications, WLANs and Broadcasting, John Wiley \& Sons, 2003, pp978.

[5] L-L Yang, L. Hanzo, Serial Acquisition Performance of SingleCarrier and Multicarrier DS-CDMA over Nakagami-m Fading Channels, IEEE Transactions on Wireless Communications, vol. 1, No.4, Issue 4, 2002, pp692-702.

[6] S.H. Won, L. Hanzo, Initial and Post-Initial Acquisition in the Serial Search Based Noncoherent Multiple Transmit/Receive Antenna Aided DS-CDMA Downlink, Vehicular Technology Conference, 7 10 May 2006, Melbourne, Australia, CD-ROM.

[7] J-C Lin, Differentially Coherent PN Code Acquisition with FullPeriod Correlation in Chip-Synchronous DS/SS Receivers, IEEE Transactions on Communications, vol. 50, No.5, Issue 5, 2002, pp698-702.

[8] A.J. Viterbi, CDMA: Principles of Spread Spectrum Communication, Chapter 3, Addison-Wesley, 1995, pp39-75.

[9] J.G. Proakis, Digital Communications, 4th ed. Chapter 2, McGrawHill, 2001, pp17-79.

[10] H. Holm, M-S. Alouini, Sum and Difference of Two Squared Correlated Nakagami Variates in Connection with the McKay Distribution, IEEE Transactions on Communications, vol. 52, No.8, Issue 8, 2004, pp1367-1376.

[11] H-F Lu, P.V. Kumar, Rate-Diversity Tradeoff of Space-Time Codes With Fixed Alphabet and Optimal Constructions for PSK Modulation, IEEE Transactions on Information Theory, Vol. 49, No. 10, Issue 10, 2003, pp2747-2751.

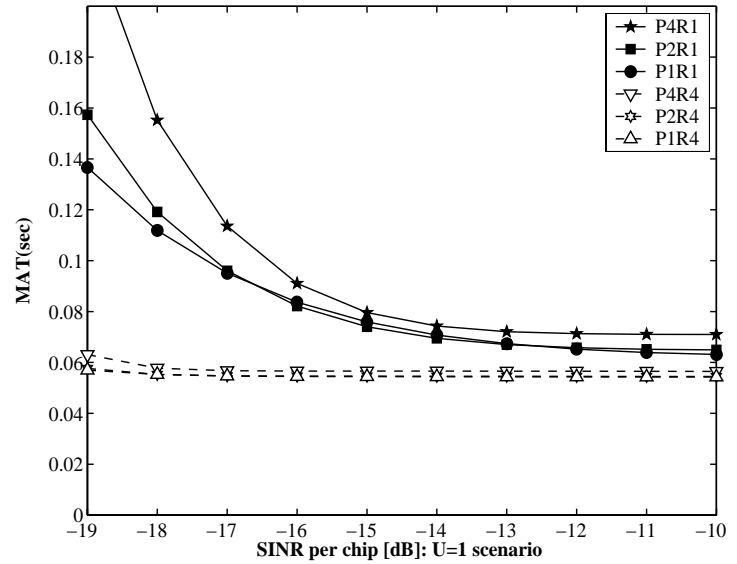

Fig. 3. MAT versus SINR per chip performance of the SDSS-aided differentially coherent code acquisition scheme parameterised with both the number of transmit and receive antennas for $U=1$ subcarriers.

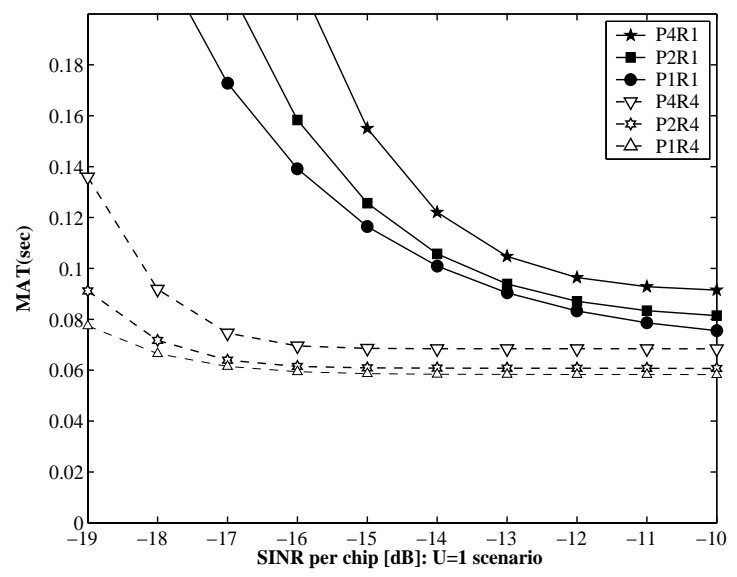

Fig. 4. MAT versus SINR per chip performance of the SDSS-aided noncoherent code acquisition scheme parameterised with both the number of transmit and receive antennas for $U=1$ subcarriers.

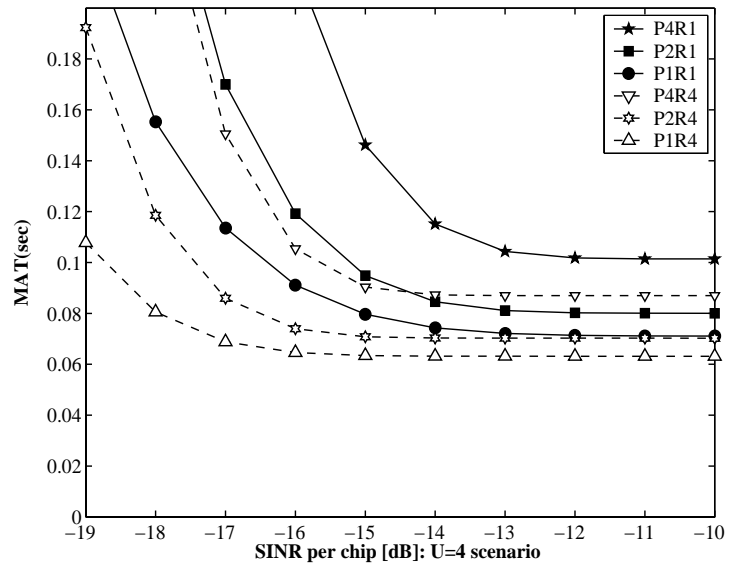

Fig. 5. MAT versus SINR per chip performance of the SDSS-aided differentially coherent code acquisition scheme parameterised with both the number of transmit and receive antennas for $U=4$ subcarriers.

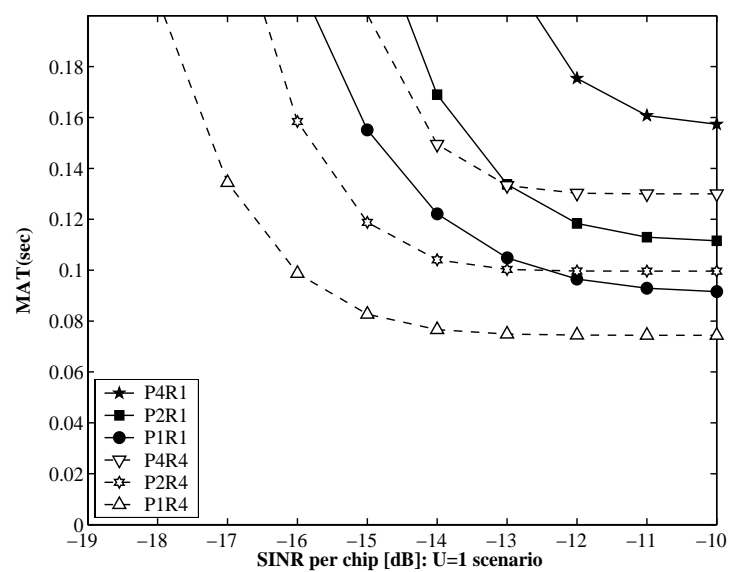

Fig. 6. MAT versus SINR per chip performance of the SDSS-aided noncoherent code acquisition scheme parameterised with both the number of transmit and receive antennas for $U=4$ subcarriers. 